Ferrata Storti Foundation

\title{
Lysine specific demethylase 1 inactivation enhances differentiation and promotes cytotoxic response when combined with all-trans retinoic acid in acute myeloid leukemia across subtypes
}

Haematologica 2018

Volume 104(6):1156-1167

\section{Correspondence: \\ HELAI P.MOHAMMAD \\ helai.x.mohammad@gsk.com \\ Received: June 4, 2018. \\ Accepted: November 30, 2018. \\ Pre-published: December 4, 2018.}

doi:10.3324/haematol.2018.199190

Check the online version for the most updated information on this article, online supplements, and information on authorship \& disclosures: www.haematologica.org/content/104/6/1156

(C)2019 Ferrata Storti Foundation

Material published in Haematologica is covered by copyright. All rights are reserved to the Ferrata Storti Foundation. Use of published material is allowed under the following terms and conditions:

https://creativecommons.org/licenses/by-nc/4.0/legalcode. Copies of published material are allowed for personal or internal use. Sharing published material for non-commercial purposes is subject to the following conditions:

https://creativecommons.org/licenses/by-nc/4.0/legalcode, sect. 3. Reproducing and sharing published material for commercial purposes is not allowed without permission in writing from the publisher.
Kimberly N. Smitheman, ${ }^{1}$ Tesa M. Severson, ${ }^{2}$ Satyajit R. Rajapurkar, ${ }^{1}$ Michael T. McCabe, ${ }^{1}$ Natalie Karpinich, ${ }^{1}$ James Foley, ${ }^{1}$ Melissa B. Pappalardi, ${ }^{1}$ Ashley Hughes, ${ }^{3}$ Wendy Halsey, ${ }^{3}$ Elizabeth Thomas, ${ }^{3}$ Christopher Traini, ${ }^{3}$ Kelly E. Federowicz, ${ }^{1}$ Jenny Laraio, ${ }^{1}$ Fredrick Mobegi, ${ }^{2}$ Geraldine Ferron-Brady, ${ }^{4}$ Rabinder K. Prinjha, ${ }^{1}$ Christopher L. Carpenter, ${ }^{1}$ Ryan G. Kruger, ${ }^{1}$ Lodewyk Wessels ${ }^{2,5}$ and Helai P. Mohammad ${ }^{1}$

${ }^{1}$ Epigenetics Discovery Performance Unit, Oncology R\&D, GlaxoSmithKline, Collegeville, PA, USA; '2Division of Molecular Carcinogenesis, Oncode Institute, the Netherlands Cancer Institute, Amsterdam, the Netherlands; ${ }^{3}$ Target Sciences, GlaxoSmithKline, Collegeville, PA, USA; ${ }^{4}$ Clinical Pharmacology and Modeling Sciences, GlaxoSmithKline, Collegeville, PA, USA and ${ }^{5}$ Faculty of EEMCS, Delft University of Technology, the Netherlands

\section{ABSTRACT}

L ysine specific demethylase 1 (LSD1) is a histone modifying enzyme that suppresses gene expression through demethylation of lysine 4 on histone H3. The anti-tumor activity of GSK2879552 and GSK-LSD1, potent, selective irreversible inactivators of LSD1, has previously been described. Inhibition of LSD1 results in a cytostatic growth inhibitory effect in a range of acute myeloid leukemia cell lines. To enhance the therapeutic potential of LSD1 inhibition in this disease setting, a combination of LSD1 inhibition and all-trans retinoic acid was explored. All-trans retinoic acid is currently approved for use in acute promyelocytic leukemia in which it promotes differentiation of abnormal blast cells into normal white blood cells. Combined treatment with all-trans retinoic acid and GSK2879552 results in synergistic effects on cell proliferation, markers of differentiation, and, most importantly, cytotoxicity. Ultimately the combination potential for LSD1 inhibition and ATRA will require validation in acute myeloid leukemia patients, and clinical studies to assess this are currently underway.

\section{Introduction}

Acute myelocytic leukemia (AML) is characterized by excessive growth of hematopoietic progenitor cells that reach varying stages of differentiation depending on the subtype. With the exception of acute promyelocytic leukemia (APL) few patients with AML are cured, despite treatment that includes high-dose induction and consolidation therapy and even, for some, bone marrow transplant. ${ }^{1}$ The disease is classified using the French-American-British (FAB) classification that divides AML into eight subtypes (M0 to M7) based on the differentiation status of the tumor cells as well as the cell type from which the cancer arises. The World Health Organization (WHO) further distinguishes AML types by also considering somatic genetic alterations. ${ }^{2}$ For most subtypes, first-line treatment consists of chemotherapy followed, in some instances, with hematopoietic stem cell transplant (HSCT). ${ }^{3}$ Due to the intensity of HSCT treatment, this approach is often only recommended for younger patients or those deemed fit enough to tolerate it. Even among the younger patient population, the 5-year overall survival is only approximately $40 \% .{ }^{3}$ For patients over the age of 60 , only approximately $20 \%$ survive; ${ }^{4}$ therefore, more effective second-line treatment options are needed.

Lysine specific demethylase 1 (LSD1) is a histone-modifying enzyme that is a member of the monoamine oxidase family. ${ }^{5}$ LSD1 has been shown to suppress 
gene expression through demethylation of mono and dimethyl groups present on lysine 4 of histone H3. ${ }^{6}$ LSD1 is a critical regulator of hematopoiesis, in part, through interaction with the transcription factors GFI-1 and GFI1b. This LSD1-containing complex regulates expression of key myeloid differentiation genes and ultimately controls hematopoietic progenitor cell differentiation. ${ }^{7}$ LSD1 is frequently over-expressed in human cancers, including AML, and knockdown of LSD1 has been shown to inhibit the growth of AML cells. ${ }^{1,8-10}$ These data have spurred interest in LSD1 as a potential target for treatment of AML. As previously reported, potent, selective, irreversible inactivators of LSD1 have been developed, and among the cancer cell lines evaluated, these show selective anti-proliferative activity in SCLC and AML cell lines. ${ }^{9,11-13}$ Preclinical data such as these have led to the clinical development of LSD1 inhibitors in relapsed, refractory AML patients.

To build upon the therapeutic potential of LSD1 inhibition in AML, rational combination hypotheses and combinations with standard of care agents were considered. All-trans retinoic acid (ATRA) is used clinically to treat acute promyelocytic leukemia (APL), a subtype of AML, and has been shown to be hugely successful, achieving curative effects in this disease subtype. ${ }^{14}$ ATRA triggers the transcription factor retinoic acid receptor alpha $(\mathrm{RAR} \alpha)$ to bind to retinoic acid response elements found in the genome and initiate transcription of target genes, including those important for differentiation. ${ }^{15}$ APL is characterized by a PML-RAR $\alpha$ fusion that inactivates RAR $\alpha$ by preventing it from its normal binding and thus locking the tumor in an undifferentiated state. ATRA degrades this fusion, allowing RAR $\alpha$ to activate its target genes, leading to differentiation and apoptosis of the cancer cells. ${ }^{16,17}$ Many clinical trials have attempted to extend the use of ATRA into non-APL AML, but unfortunately these have demonstrated very little success. ${ }^{18}$ Since the discovery of LSD1 and the characterization of its role in hematopoiesis, there has been speculation as to the possibility of combining an inhibitor of LSD1 with ATRA. One report demonstrated that combination of ATRA with knockdown of LSD1 or tranylcypromine, a nonselective monoamine oxidase inhibitor with weak LSD1 inhibitory activity, leads to transcriptional activation of many RAR target genes that normally lack methylation of H3K4me2 at their promoters. ${ }^{19,20}$ This combination also had more robust anti-leukemic activity than either treatment alone in the model tested. ${ }^{19}$

The current report demonstrates the synergistic activity of a combination of a selective, potent inhibitor of LSD1, GSK2879552, with ATRA, and characterizes the mechanism associated with this combination. As a single agent, LSD1 inhibition promotes differentiation of AML cell lines and synergistic differentiation activity is observed when used in combination with ATRA across AML subtypes. This combination also enhances LSD1 inhibitormediated growth inhibition of AML cell lines and primary patient samples. Most importantly, treatment of AML cell lines with an LSD1 inhibitor and ATRA results in synergistic cytotoxicity and caspase-mediated cell death. Collectively, these data suggest that this combination may result in greater efficacy and may be more likely to translate to response in AML patients than LSD1 inhibitor monotherapy. Moreover, this combination may expand the therapeutic potential of ATRA, a proven ther- apy, to non-APL AML patients. Clinical studies are currently underway to test this hypothesis in relapsed, refractory AML patients (clinicaltrials.gov identifiers: 02273102, 02717884, 02842827).

\section{Methods}

\section{Cell lines and human biological samples}

Cell lines were obtained from the American Type Culture Collection (ATCC) or the Deutsche Sammlung von Mikroorganismen und Zellbulturen (DSMZ). Human biological samples were sourced ethically and informed consent was obtained for their use in research. The use of human tissue samples was reviewed and approved by the GSK Research \& Development Compliance (RDC) Human Biological Sample Use Committee.

\section{Compounds}

GSK2879552 and GSK-LSD1 (GlaxoSmithKline), ATRA (Sigma), and Bortezomib (Chemietek) were prepared in 100\% dimethyl sulfoxide (DMSO) (Sigma).

\section{Cell proliferation}

Proliferation assays were conducted as previously described. ${ }^{11}$ The growth-death index (GDI) value was determined as the percentage of cells relative to end-of-assay vehicle and assay start, where the number of cells in the vehicle is set at $100 \%$ and the number of cells at the time of compound addition $\left(T_{0}\right)$ is set to $0 \%$. A minimum of two biological replicates were evaluated for each assay. Bliss Independence analysis was performed, and a synergy score was determined. ${ }^{9,21}$

\section{Mechanistic and phenotypic assays}

A minimum of two biological replicates were evaluated for each assay. Dose response curves were generated using a 4-parameter equation (XLfit, IDBS). All kits were used according to the manufacturer's recommendations. Cell cycle phase distribution was determined by flow cytometry using the Cycletest ${ }^{\mathrm{TM}}$ PLUS DNA Reagent Kit (BD Biosciences) and data were analyzed using FlowJo software (TreeStar Inc.). Proliferation was assessed using BrdU Cell Proliferation Assay Kit (Cell Signaling Technology) with a 4-hour pulse. Values were expressed as percent of vehicle. Caspase activity was measured using Caspase-Glo 3/7 (Promega). Luminescence values were normalized to CTG (Promega) for cell number. Peak activation was determined for MOLM-13, OCIAML3, MV-4-11, THP-1, SIG-M5, HL-60, and Kasumi-1 to be days $4,6,6,3,4,5$, and 5 , respectively. Values were expressed as a fold change relative to vehicle. Gene expression was evaluated using real time-quantitative polymerase chain reaction (RT-qPCR), as previously described. ${ }^{11}$ Superoxide anion production was measured using LumiMax Superoxide Anion Detection Kit (Agilent Technologies). Values were expressed as fold increase over vehicle. Morphology was visually assessed after staining with MayGrunwald and Giemsa (Sigma).

\section{Flow cytometry}

Cells were stained with surface marker antibodies (CD11b, 555388 or 557754; CD86, 555657; CD71, 555537) and 7-AAD obtained from BD Biosciences. Percent positive for each marker was determined relative to isotype control from cells that were 7$\mathrm{AAD}$ negative. Additionally, cells were stained with the above cocktail including annexin V (BD Biosciences) to evaluate Annexin $\mathrm{V}$ positivity. A minimum of two biological replicates were evaluated for each assay. 
A

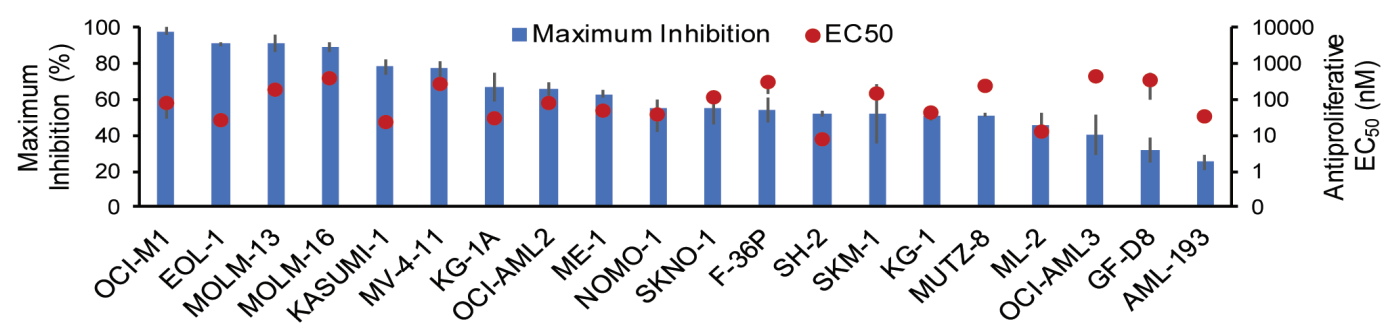

B

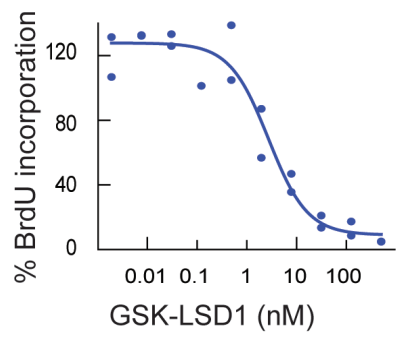

D

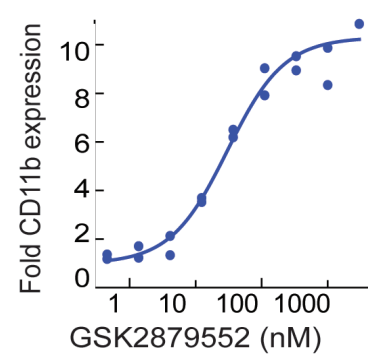

F

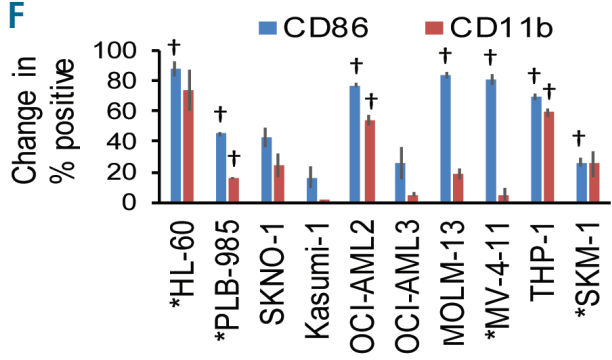

H
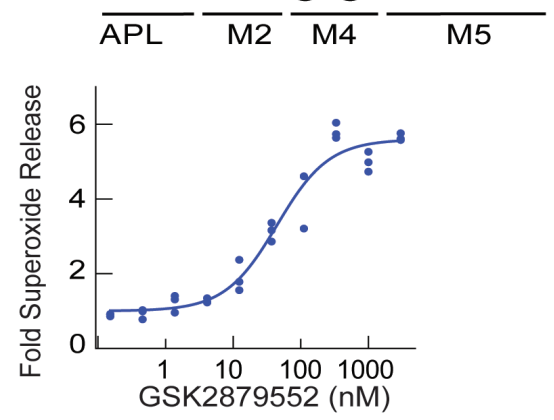

C

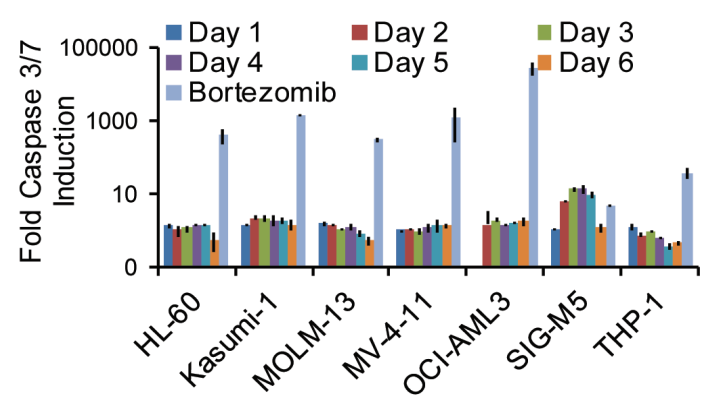

E

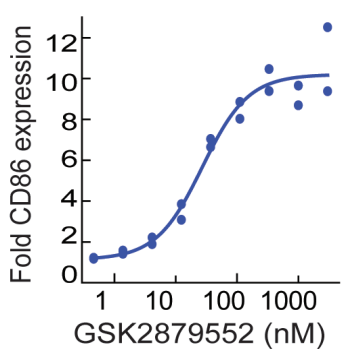

G
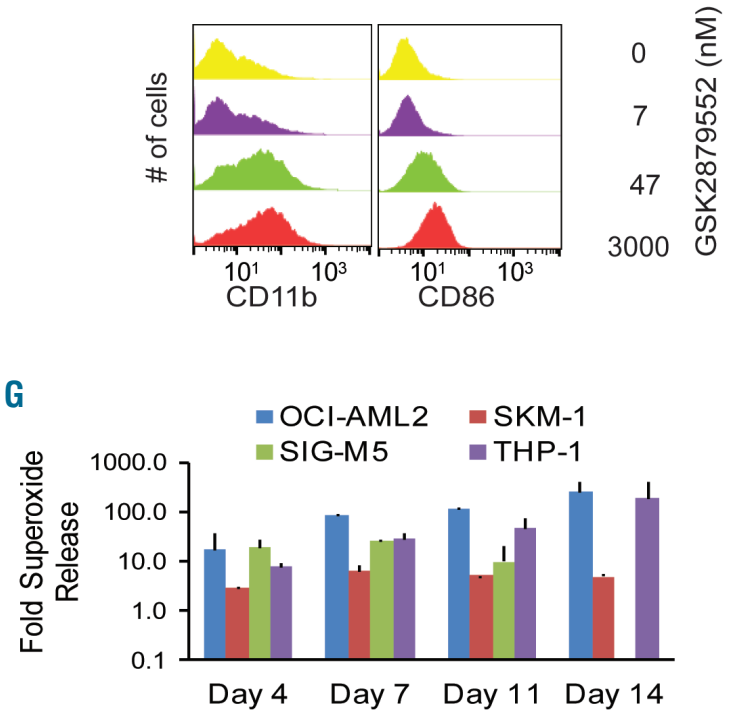

I

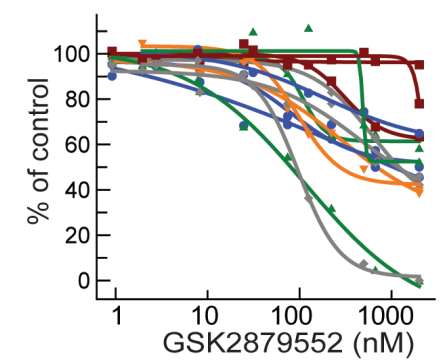

Figure 1. Inactivation of LSD1 inhibits the growth of acute myeloid leukemia (AML) cells by releasing myeloid differentiation block. (A) Percent maximum inhibition (bars) \pm Standard Error and $\mathrm{EC}_{50}$ (circles) for 20 human AML cell lines treated with a titration of GSK2879552 for ten days. (B) Dose response of incorporation by BrdU Cell Proliferation Assay Kit (Cell Signaling Technology) of MOLM-13 cells treated with a titration of GSK-LSD1 for six days. (C) Fold caspase 3/7 activation ( \pm Standard Error) relative to vehicle control of AML cell lines treated with $1000 \mathrm{nM}$ GSK2879552 for 1-6 days or 100 nM bortezomib for three days. (D) Dose response of ITGAM (CD11b, left) and CD86 (right) gene expression on MOLM-13 cells treated with a titration of GSK2879552 for one day. (E) Representative flow cytometric histograms of CD11b protein expression on THP-1 cells (left) and CD86 protein expression on MOLM-13 cells (right) treated with GSK2879552 for one day. Concentrations of GSK2879552 from top to bottom are 0, 7, 47, and $3000 \mathrm{nM}$. (F) The change (treatment-vehicle) in the percentage of cells positive for CD86 (blue) and CD11b (red) protein expression ( \pm Standard Error) for ten AML cell lines treated with 500 nM GSK2879552 (or 1000 nM GSK2879552*) for three days. †Significance $(P<0.05)$ between dimethyl sulfoxide (DMSO) and GSK552 treatment. (G) Peak superoxide anion production ( \pm Standard Error) of four AML cell lines treated with 1 MM GSK2879552 for 4, 7, 11, and 14 days. (H) Peak superoxide anion production of SKM-1 cells treated with a titration of GSK2879552 for seven days. (I) Dose responses of AML blast colony formation of 14 patient samples treated with a titration of GSK2879552. APL: acute promyelocytic leukemia. 


\section{Primary acute myeloid leukemia blast colony}

Clonogenic progenitors from primary AML patients' samples were assessed in a semi-solid methylcellulose-based medium (ColonyGel $^{\mathrm{TM}}$, ReachBio) containing recombinant human (rh) SCF (50 ng/mL), rhIL-3 (10 ng/mL), rhGM-CSF (10 ng/mL), and rhEpo ( $3 \mathrm{U} / \mathrm{mL}$ ). Each concentration of compound was tested with three technical replicates. Values were expressed as percent of vehicle and graphed as dose response curves (XLfit, IDBS). All known patients' sample characteristics are provided in Online Supplementary Table S1A.

\section{RNA-sequencing}

Methods for RNA-sequencing can be found in the Online Supplementary Appendix.

\section{Results}

Lysine specific demethylase 1 inhibition reduces growth and promotes differentiation of acute myeloid leukemia cells

To investigate the role of LSD1 activity on the growth of AML cells, AML cell lines were treated with LSD1 inhibitors. As shown previously, GSK2879552 and a structurally similar compound, GSK-LSD1, are both potent, selective inactivators of LSD1. ${ }^{11}$ GSK2879552 was analyzed for its ability to inhibit tumor cell growth in a 10day proliferation assay using CellTiter-Glo (Figure 1A). The concentration required to achieve half of the maximal observed effect $\left(\mathrm{EC}_{50}\right)$ as well as the maximum percent
A

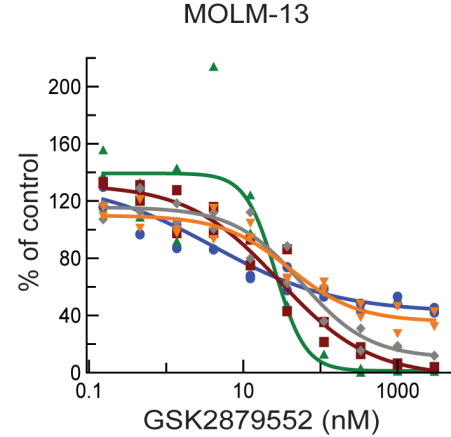

B

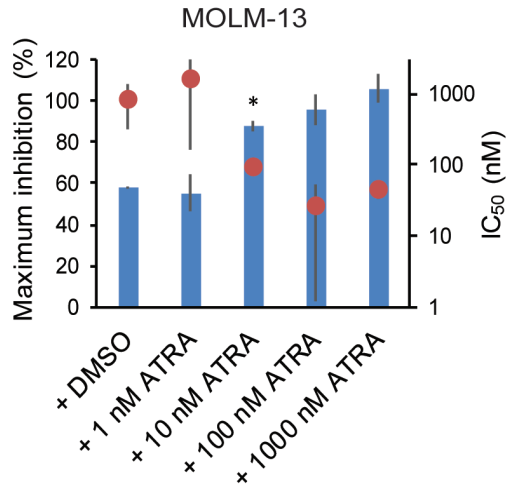

C

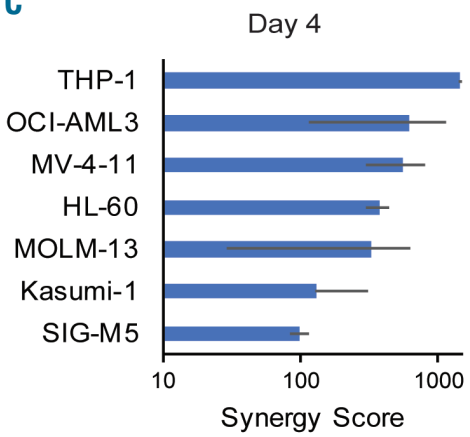

OCI-AML 3
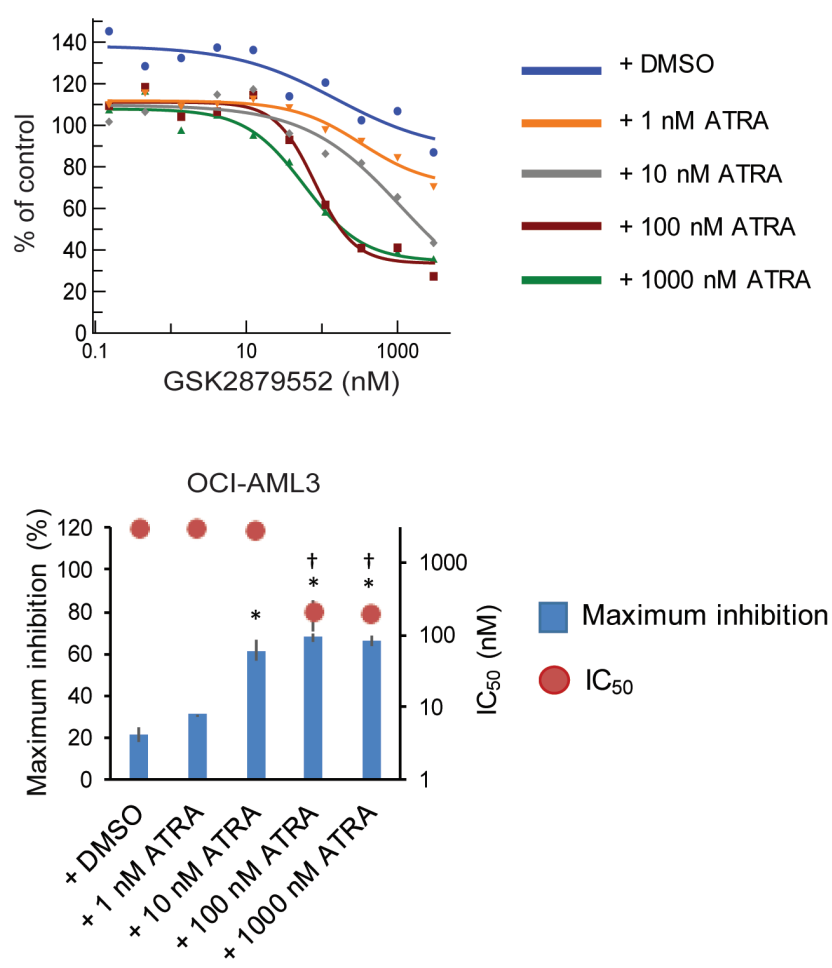

D

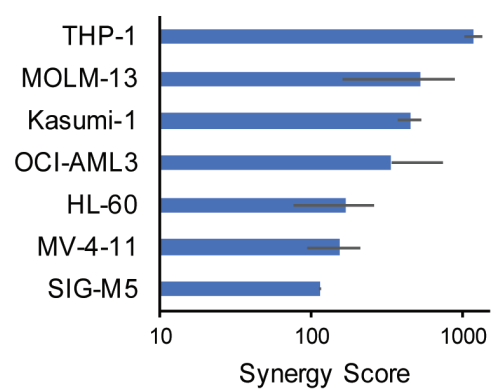

Figure 2. Sensitivity of acute myeloid leukemia (AML) cells to GSK2879552 increases with addition of all-trans retinoic acid (ATRA). (A) Dose response curves of MOLM-13 (left) and OCI-AML3 (right) cells treated with a titration of GSK2879552 $\pm 1,10,100$, or 1000 nM ATRA for four days. Values were graphed relative to the

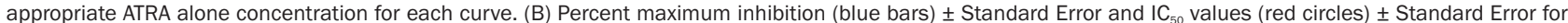
MOLM-13 (left) and OCI-AML3 (right) cells from (A). *Significance $(P<0.05)$ between dimethyl sulfoxide (DMSO) and ATRA treatments for maximum inhibition; ${ }^{\dagger}$ significance $(P<0.05)$ between dimethyl sulfoxide (DMSO) and ATRA treatments for IC50. (C) Synergy scores ( \pm Standard Error) for seven AML cell lines treated with a titration of GSK2879552 $\pm 1,10,100$, or $1000 \mathrm{nM}$ ATRA for four days. (D) Synergy scores ( \pm Standard Error) for seven AML cell lines treated with a titration of GSK2879552 $\pm 1,10,100$, or $1000 \mathrm{nM}$ ATRA for six days. 
A



OCl-AML3, Day 2

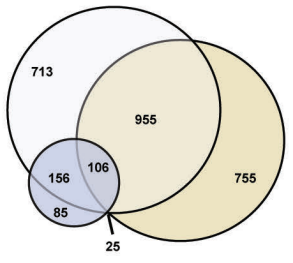

MOLM-13, Day 4

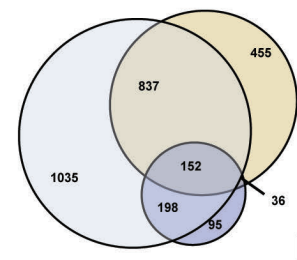

OCl-AML3, Day 4

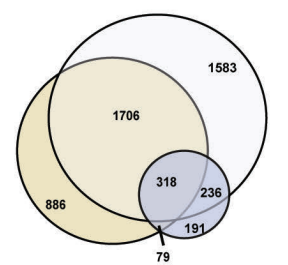

B

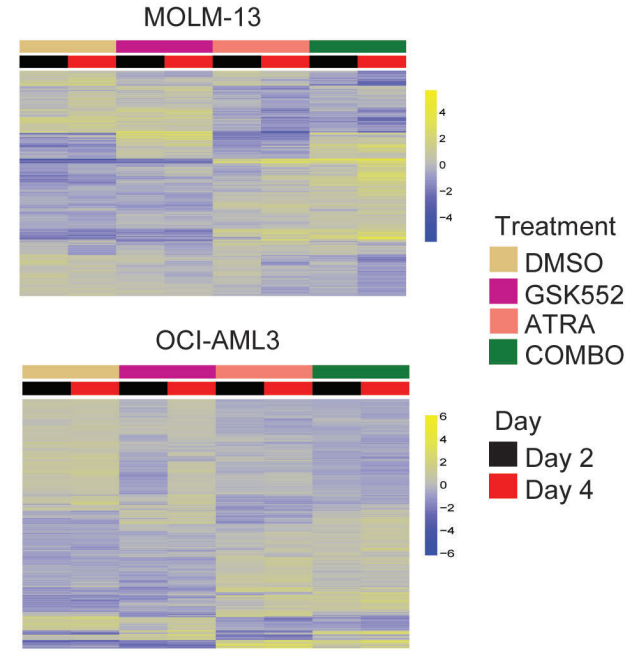

D

Day 2

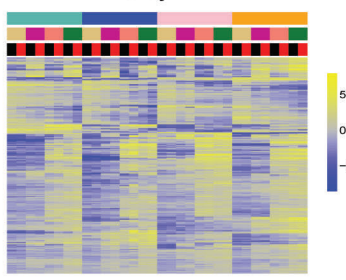

Cell Line

MOLM-13

MV-4-11

OCI-AML3

THP-1

Treatment

DMSO

GSK552

ATRA

COMBO

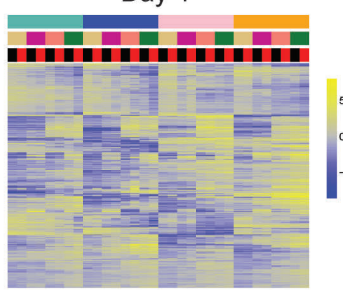

Day

Day 2

Day 4

E
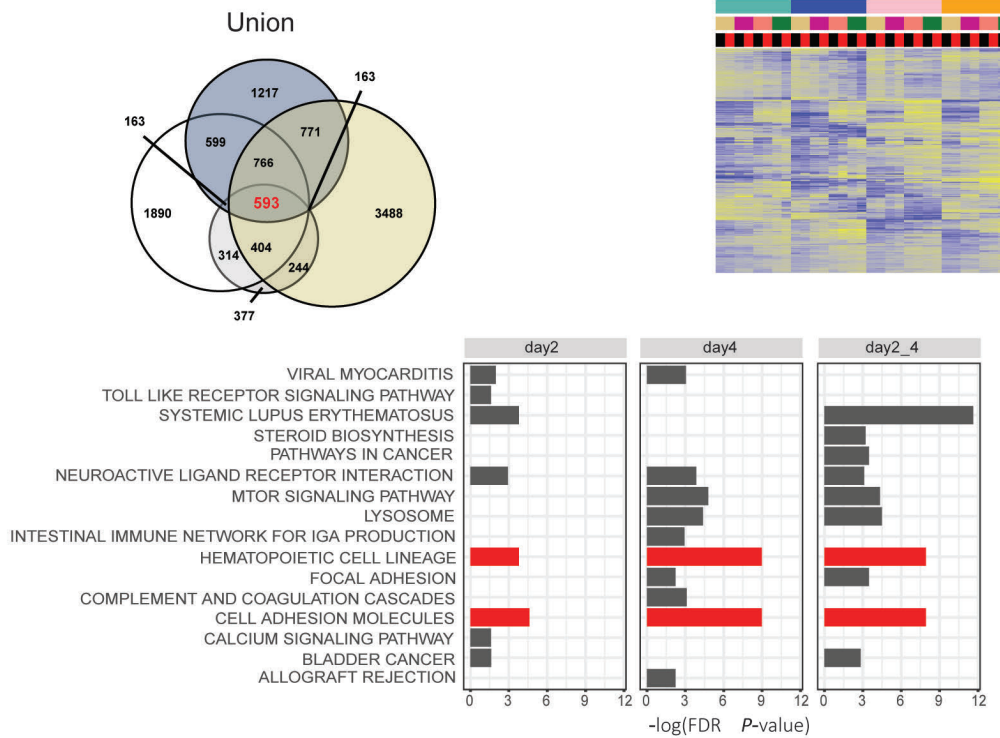

Figure 3. RNA sequencing reveals differentiation gene signature with combination (COMBO). (A) Venn diagram depicting the overlap of significantly differentially expressed genes in the specific treatments [GSK2879552 + all-trans retinoic acid (ATRA) combination relative to dimethyl sulfoxide (DMSO) (light blue), ATRA relative to DMSO (yellow), and GSK2879552 relative to DMSO (blue)] for MOLM-13 (top) and OCl-AML3 (bottom) cell lines at day 2 (left) and day 4 (right). (B) Corresponding gene expression heatmaps depicting the union of genes from day 2 and day 4 Venn diagrams (from A) for MOLM-13 (top) and OCI-AML3 (bottom) cell lines. Values are row-mean centered, $\log _{2}$ normalized, gene expression data shown high (yellow) to low (blue). (C) Venn diagrams depicting the overlap of significantly differentially expressed genes for the GSK2879552 + ATRA combination relative to DMSO in each of the four cell lines, OCIAML-3, MOLM-13, MV-4-11, and THP-1. Data for different time points are shown, day 2 (top, left), day 4 (top, right), and union of day 2 and day 4 (bottom). Red value indicates overlap of significant genes in all four cell lines for both days. (D) Gene expression heatmaps of overlapping combination versus DMSO genes in intersect (from C) for day 2 (top) and day 4 (bottom). Values are row-mean centered, $\log _{2}$ normalized, gene expression data shown high (yellow) to low (blue). (E) Barplots showing the top $10 \mathrm{KEGG}$ pathways found to be significantly enriched from genes in (C) in intersect (day 2, day 4 and union of day 2 and day 4 tested separately). Bars show the $-\log (($ False Discovery Rate $P$-value) on the $x$-axis and the MSigDB KEGG pathways identified as enriched in at least one timepoint on the $y$-axis. Red bars indicate pathways that were also identified as enriched in all four cell lines across both time points using an alternative method (Online Supplementary Figure S3D). 
inhibition were determined for each cell line. GSK2879552 inhibited cell growth with a potent average $\mathrm{EC}_{50}$ value of $137 \pm 30 \mathrm{nM}$ across 20 cell lines. The average maximum inhibition was $62 \pm 5 \%$ suggesting complete cytostasis is not achieved by ten days with LSD1 inhibition alone. By comparing 6-day to 10-day proliferation assay results (Online Supplementary Figure S1A and B), it is evident that an increase in maximum inhibition can be achieved with longer treatment, indicating that the biological consequences of LSD1 inhibition, including growth effects, may require time to be fully revealed. Several studies were conducted to determine if the lag in inhibition of cancer cell line growth is due to a delayed effect on all cells or involves some cell death. Cell cycle evaluation of MOLM13 cells treated with a titration of GSK-LSD1 for six days revealed an increase in cells in G1 phase with a concomi-
A

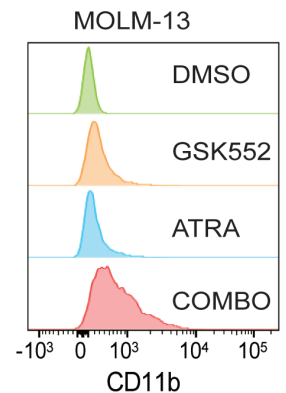

C

MOLM-13



D
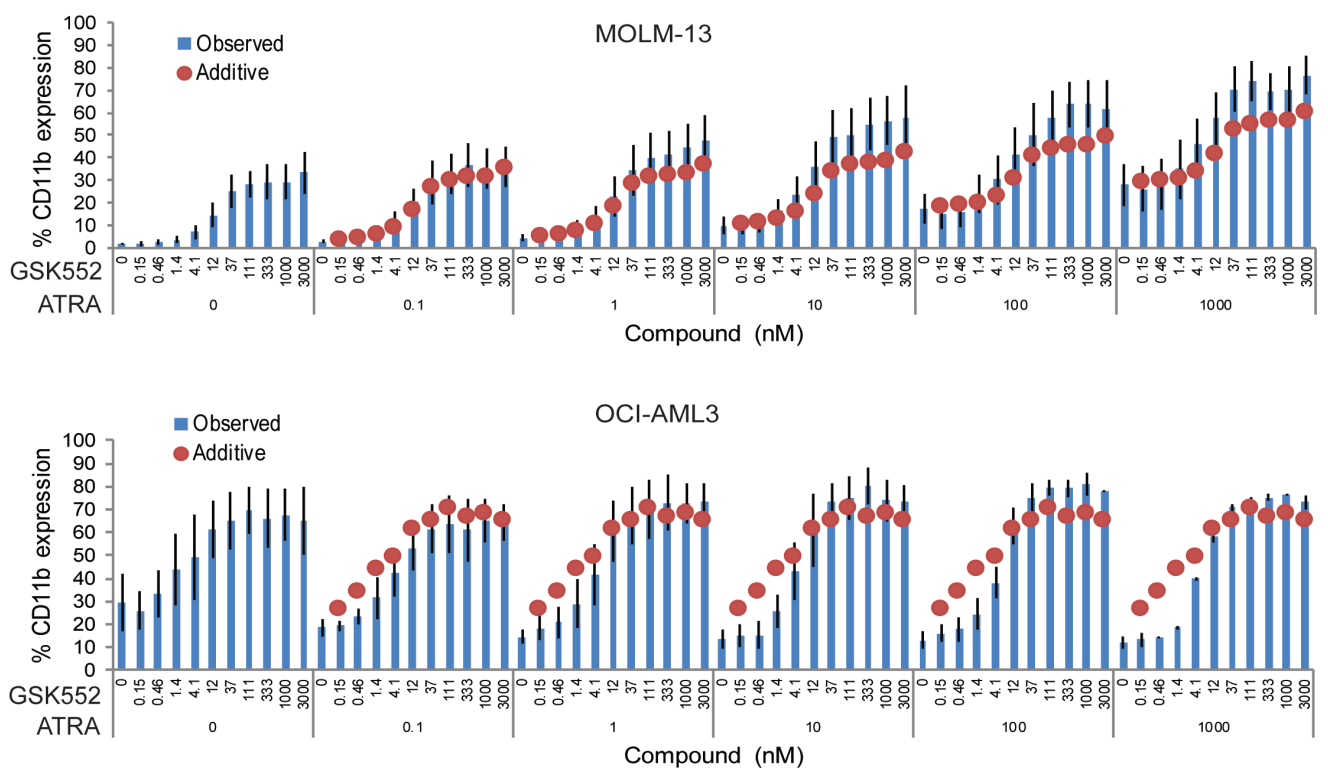

GSK2879552 (nM)

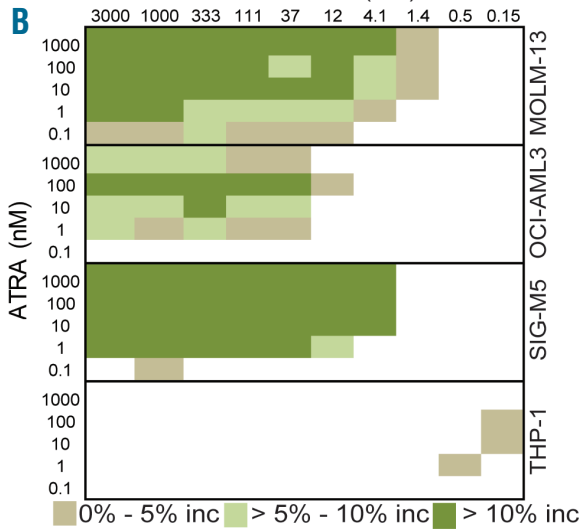

GSK552 + DMSO

GSK552 + 0.1 nM ATRA

GSK552 + 1 nM ATRA

GSK552 + 10 nM ATRA

GSK552 + 100 nM ATRA

GSK552 + 1000 nM ATRA

ATRA + DMSO

Figure 4. The addition of all-trans retinoic acid (ATRA) enhances GSK2879552 induced differentiation effects on acute myeloid leukemia (AML) cells. (A) Representative histograms of CD11b surface marker expression on MOLM-13 cells (left) and OCl-AML3 cells (right) treated with dimethyl sulfoxide (DMSO) (green), 1000 nM GSK2879552 (orange), 100 nM ATRA (blue), or 1000 nM GSK2879552 plus 100 nM ATRA (red) for two days. (B) Dose response curves of CD11b surface marker expression on MOLM-13 cells (left) and OCI-AML3 cells (right) treated with a titration of GSK2879552 + 0, 0.1, 1, 10, 100, or 1000 nM ATRA for two days. (C) Percent $\mathrm{CD} 11 \mathrm{~b}^{+}$expression (blue bars, \pm Standard Error) on MOLM-13 cells (top) and OCl-AML3 cells (bottom) treated with the indicated concentrations of GSK2879552 and ATRA for two days. The value representing the predicted additive effect at each combination concentration is indicated with a red circle. (D) Shading represents percent CD11 $\mathrm{b}^{+}$expression relative to additive threshold on AML cells treated with the indicated concentrations of GSK2879552 and ATRA for two days. Dark green shading indicates $>10 \%$ above additive threshold, light green indicates $>5-10 \%$ above additive threshold, and light brown indicates $0-5 \%$ above additive threshold. 
tant decrease in S phase (Online Supplementary Figure S1C). No appreciable accumulation of cells in sub-G1 was observed. To confirm the cell cycle findings, a BrdU assay that measures actively dividing cells by incorporation of BrdU into DNA during S-phase was performed (Figure 1B). MOLM-13 cells treated for six days had a dose responsive decrease in $\mathrm{BrdU}$ signal $\left(\mathrm{EC}_{50}=1.9 \pm 0.9 \mathrm{nM}\right)$. Finally, induction of caspase 3/7 was assessed to determine if cells were activating the apoptosis pathway. Seven AML cell lines were treated for up to six days with GSK2879552. In six of seven AML cell lines there was no appreciable induction of caspase 3/7 at any time point
A

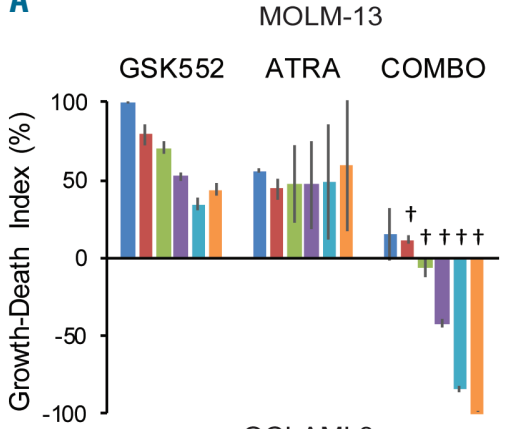

OCI-AML3

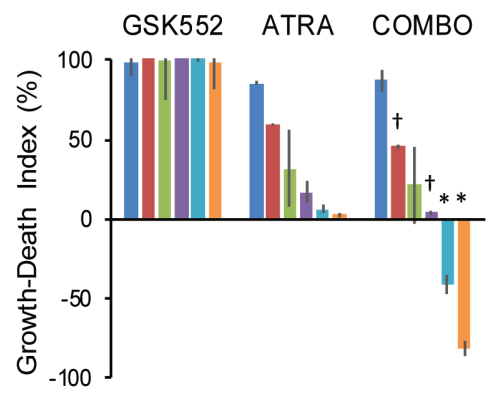

C


day 1

day 2

day 3

day 4

day 5

day 6

day 1

day 2

day 3

day 4

day 5

day 6

B

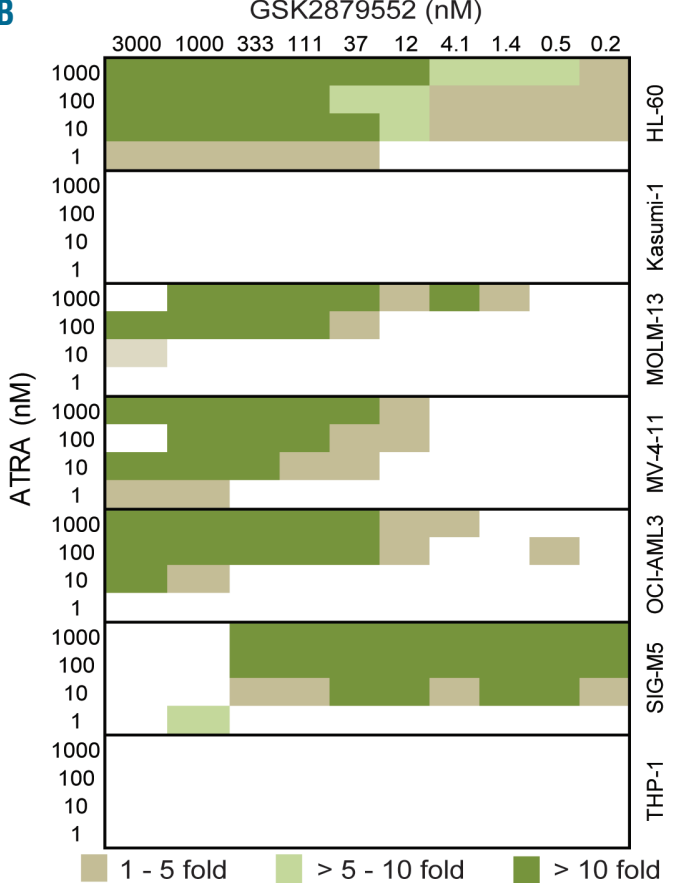

MOLM-13

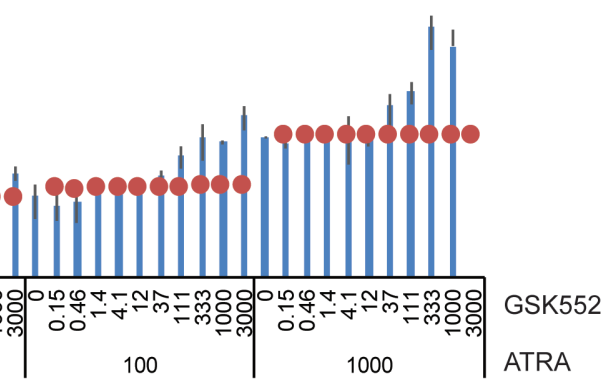

Compound (nM)

OCI-AML3

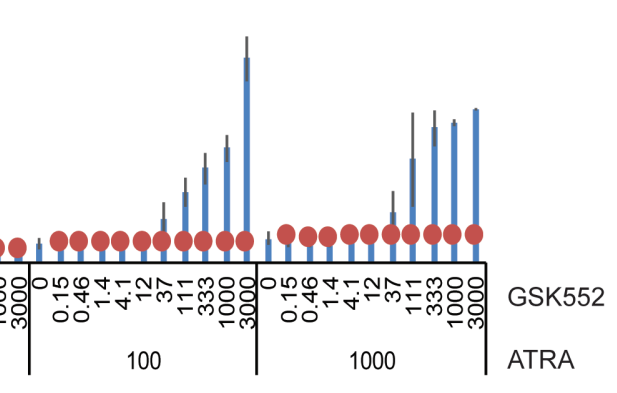

Compound (nM)

Figure 5. The combination of GSK2879552 and all-trans retinoic acid (ATRA) promotes caspase-mediated cell death. (A) GDI values ( \pm Standard Error) of MOLM13 (left) and OCl-AML3 (right) cells treated with 1000 nM GSK2879552, 100 nM ATRA, or 1000 nM GSK2879552 + 100 nM ATRA (COMBO) for 1 to 6 days. 'Significance $(P<0.05)$ between GSK552 and COMBO. * Significance $(P<0.05)$ between GSK552 and COMBO as well as between ATRA and combination $($ COMBO). (B) Fold caspase $3 / 7$ cleavage values from day of peak induction (blue bars, \pm Standard Error) for MOLM-13 cells (top) and OCl-AML3 cells (bottom) treated with the indicated concentrations of GSK2879552 and ATRA. The value representing the additive effect at each combination concentration is indicated with a red circle. (C) Shading represents fold caspase 3/7 cleavage values relative to threshold from day of peak induction of AML cells treated with the indicated concentrations of GSK2879552 and ATRA. Dark green shading indicates >10-fold above additive threshold, light green indicates 5- to 10-fold above additive threshold, light brown indicates 1- to 5 -fold above additive threshold. 
(Figure 1C). These data indicate that the growth inhibitory effects of LSD1 inhibitors are due to a slowing of cell division and not significant killing of tumor cells.

Lysine specific demethylase 1 is a known regulator of normal hematopoiesis whereby it maintains hematopoietic stem and progenitor cell populations in a quiescent state through GFI1-mediated transcriptional repression of HoxA9 and Meis1.22 Additionally, several reports have shown that LSD1 inhibition promotes differentiation of leukemic cells. ${ }^{10}$ Knockdown of LSD1 leads to an increase in markers of differentiation, and this effect has been recapitulated using small molecule inhibitors of LSD1.,12 To determine if growth inhibition achieved with GSK2879552 occurs in association with cellular differentiation, MOLM-13 cells were treated for one day with a titration of GSK2879552, and expression of myeloid differentiation marker genes, ITGAM (CD11b) and CD86, was measured by RT-PCR (Figure 1D). Higher expression of $\mathrm{CD} 11 \mathrm{~b}$ is indicative of differentiation from an immature myelo/monoblast to a more mature myeloid cell, and CD86 is co-expressed with CD80 on mature macrophages and dendritic cells where it is poised to interact with $\mathrm{CD} 4^{+}$ $T$ cells. 23,24 GSK2879552 increased expression of CD11b and $\mathrm{CD} 86$ genes with average $\mathrm{EC}_{50}$ values of $31 \pm 1 \mathrm{nM}$ and $28 \pm 6 \mathrm{nM}$, respectively. To determine if these increases in gene expression translate to increased protein levels, cell surface expression of CD11b and CD86 were measured in THP-1 and MOLM-13 cells by flow cytometry (Figure 1E and Online Supplementary Figure S1D and E). The percent of cells positive for each surface marker was determined by gating against an isotype control. Cells treated with a titration of GSK2879552 for one day showed a dosedependent increase in protein expression with average EC50 values of $23 \pm 4 \mathrm{nM}$ and $44 \pm 4 \mathrm{nM}$ for THP- 1 and MOLM-13 cells, respectively.

To determine if the effect of GSK2879552 on differentiation markers occurs across cell lines that represent a range of AML subtypes, a panel of cell lines was evaluated by flow cytometry after treatment with either GSK2879552 or GSK-LSD1 (Figure 1F). Thirteen of 16 AML cell lines tested had at least a 10\% increase in CD86 after three days of treatment, while 10 of 16 had at least a $10 \%$ increase in $\mathrm{CD} 11 \mathrm{~b}$. Increases for both markers occurred in all five subtypes tested. Overall, the lowest increases in CD11b and CD86 occurred in the M6 subtype-derived AML cell lines (Online Supplementary Figure $S 1 F)$. This is not surprising given that M6 AMLs are erythroleukemias, and differentiation of this subtype, similar to hematopoietic progenitor cells that undergo erythopoiesis to become mature red blood cells, may not involve increases in expression of CD11b or CD86. ${ }^{22}$ To better understand the effects on M6 subtype AML cells, another marker of hematopoietic progenitor cells, transferrin receptor (CD71), was evaluated. ${ }^{25} \mathrm{CD} 71$ decreases as progenitor cells mature and, indeed, five of six M6 subtype AML cell lines showed at least a 10\% decrease in expression of CD71 after three days of treatment with GSK2879552 (Online Supplementary Figure S1F).

One characteristic of active macrophages and neutrophils is their ability to produce superoxide anion in response to stimulation; ${ }^{26,27}$ therefore, the ability of GSK2879552 to induce superoxide anion production was evaluated as a functional readout of differentiation. Four AML cell lines were treated with GSK2879552 for 4, 7, 11, and 14 days. Upon stimulation with PMA, all four cell lines released higher levels of superoxide anion when pretreated with LSD1 inhibitor (range: 3- to 249-fold over DMSO treated cells) (Figure 1G). SKM-1 cells were treated with a titration of GSK2879552 for seven days, and upon PMA stimulation, superoxide anion production was induced with an $\mathrm{EC}_{50}$ of $222 \pm 103 \mathrm{nM}$ (Figure $1 \mathrm{H}$ ).

To determine if the anti-proliferative effect of LSD1 inhibition in AML cell lines translates to primary AML, patient-derived samples that had not been subjected to long-term cell culture were evaluated using a blast colony formation assay. The number of blast colony forming units was enumerated after treatment with a titration of GSK2879552 in 14 AML patient samples. Twelve of the 14 samples were sensitive, defined as $\geq 30 \%$ inhibition, to GSK2879552 (Figure 1I). The majority of samples showed reduced colony growth by less than $50 \%$ while two samples reached complete reduction in blast colonies at the highest dose tested. These data suggest that the antitumor activity of GSK2879552 is not limited to cell lines and extends to AML patient-derived cells.

\section{The combination of LSD1 inhibitor with all-trans retinoic acid enhances anti-tumor activity}

Given that the effects of LSD1 inhibition alone on AML cells are largely cytostatic and, therefore, may not be sufficient to combat the disease clinically, efforts were made to explore combinations that could improve outcome. Previous reports suggested the combination potential of LSD1 inhibition and ATRA in non-APL AML. ${ }^{19,12}$ ATRA is used clinically for the treatment of APL and works by degrading the PML-RAR $\alpha$ fusion, resulting in differentiation of blast cells and elimination of disease. ${ }^{16}$ Schenk et al. have shown enhanced reduction of cell growth when tranylcypromine, a much less potent, non-selective inhibitor of LSD1, is combined with ATRA. ${ }^{19,20}$ Therefore, studies were undertaken to determine if a similar combination effect could be observed with the selective LSD1 inhibitor, GSK2879552. Seven AML cell lines were treated with a titration of GSK2879552 + 0, 1, 10, 100, and 1000 nM ATRA and growth was monitored for up to six days. The maximum inhibition achieved with the combination increased as the ATRA concentration increased in five of seven cell lines with a corresponding decrease in the IC50 value in four of seven cell lines on day 4 (Figure $2 \mathrm{~A}$ and $\mathrm{B}$ and Online Supplementary Figure S2A). An additional cell line showed a decrease in $\mathrm{IC}_{50}$ value without a concomitant increase in maximum inhibition. Together these data indicate that the combination of GSK2879552 and ATRA results in enhanced growth inhibition of AML cell lines. To determine whether the effect observed was synergistic, a Bliss Independence model was used (Online Supplementary Table S2A and B). ${ }^{21}$ A value $\geq 100$ indicates synergy by calculating a single score that summarizes the Bliss Independence values obtained. ' Synergistic growth inhibition is evident by day 6 in all seven AML cell lines evaluated upon treatment with GSK2879552 combined with ATRA (Figure 2C-D).

Combination of GSK2879552 with all-trans retinoic acid leads to enrichment of differentiation associated gene signatures in acute myeloid leukemia cells

To elucidate the mechanism by which GSK2879552 and ATRA combine to effect tumor cell growth, an RNA sequencing study to examine differential gene expression was conducted in six AML cell lines treated with vehicle, 
GSK2879552, ATRA, or the combination of GSK2879552 plus ATRA at two different time points. For most cell lines and time points, a smaller number of gene expression changes are found in the single treatments compared to the combination (Figure $3 \mathrm{~A}$ and $\mathrm{B}$ and Online Supplementary Figure $S 3 A$ and $B$ ). In addition, a large set of genes is found exclusively differentially expressed in combination versus DMSO, indicating that there is an effect

A



C

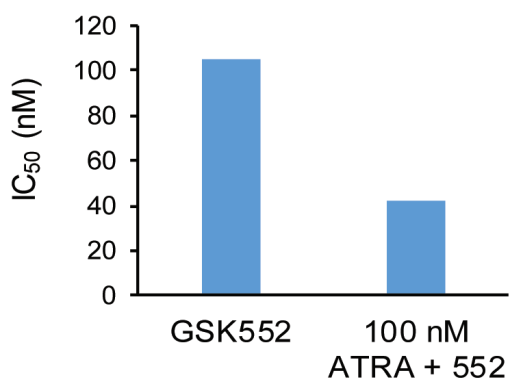

E

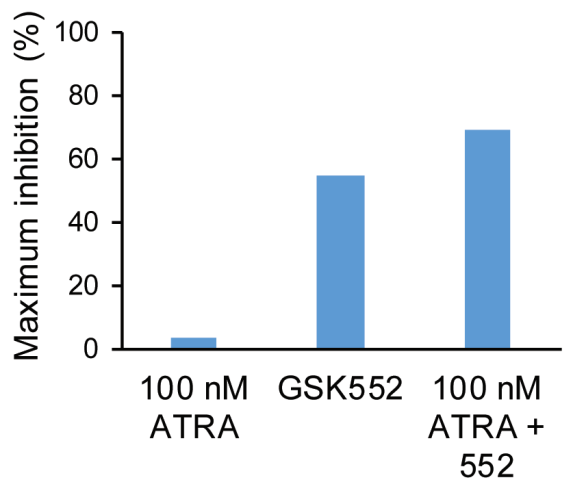

from the combination of treatments not present in the single agent treatments (Figure $\mathrm{BA}$ and $\mathrm{B}$ and Online Supplementary Figure $S 3 A$ and $B$ ). The overlap of significantly differentially expressed genes under combination treatment for each time point in four cell lines (OCIAML3, MOLM-13, MV4-11, and THP-1) is shown in Figure $3 \mathrm{C}$ (top). This indicates that a portion of genes are found in all four cell lines at each time point (intersect).

B

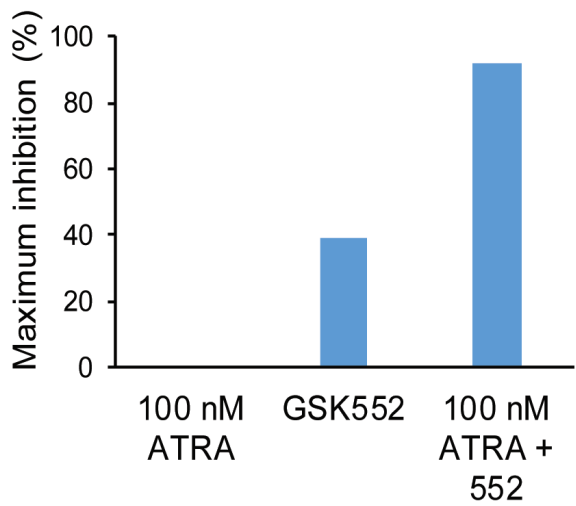

D
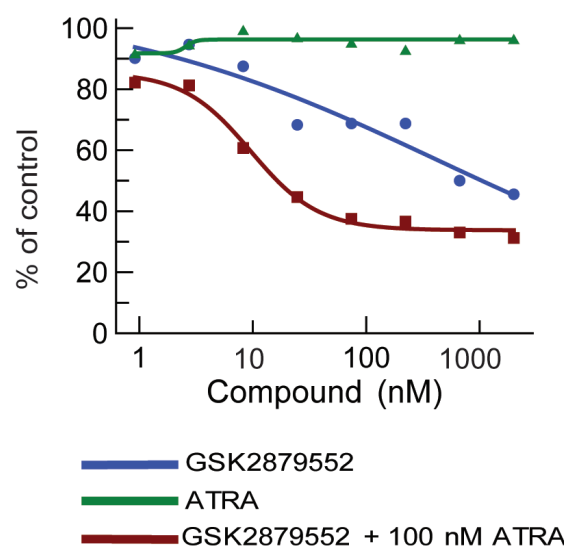

$\mathbf{F}$

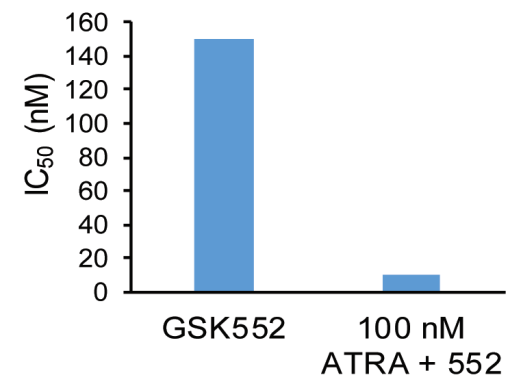

Figure 6. Acute myeloid leukemia (AML) blast colony forming ability is impaired in AML patient-derived samples treated with a combination of LSD1 inhibitor plus all-trans retinoic acid (ATRA). (A) Dose response of AML blast colony formation of patient sample AML0007 treated with a titration of GSK2879552 \pm 100 nM ATRA or a titration of ATRA. Three replicate wells per concentration were tested. (B) Maximum percent of inhibition achieved for patient sample AML0007 treated with $100 \mathrm{nM}$ ATRA or calculated from the GSK2879552 dose response curves $+100 \mathrm{nM}$ ATRA. (C) IC I0 $_{50}$ values of the GSK2879552 dose response curves \pm 100 nM ATRA for patient sample AML0007. (D-F) Same as (A-C) for patient-derived sample 4031113SH. 
The union of the time points across all four cell lines shows that 593 genes are altered in all four cell lines in response to the combination, defining an ATRA + LSD1i combination signature (Figure $3 \mathrm{C}$, bottom). The $\log _{2}$ transformed gene expression of the intersect genes for each time point (Figure 3C, top) is depicted in a heatmap that shows the row-mean centered data clustered hierarchically (Figure 3D). A subset of genes was validated by qRTPCR (Online Supplementary Figure S3C). Upon further examination of the combination treatment genes found in both time points (Figure 3C, 593 genes indicated in red) using gene set enrichment analyses, strong enrichment in KEGG pathways include hematopoietic cell lineage and cell adhesion molecule (CAMs) pathways (Figure 3E). This finding was validated using an additional methodology (Online Supplementary Figure S3D) as well as in a subset of cell lines assessed individually (Online Supplementary Figure $S 3 E)$. CAMs are involved in immune cell function and, therefore, their expression is consistent with a more differentiated state. Together with the enriched expression of genes in the hematopoietic cell lineage pathway, enrichment in CAMs through the combined treatment of GSK2879552 and ATRA suggests enhanced differentiation of AML cells.

Gene set enrichment analyses revealed additional pathways in the combination that are associated with immune cell function. The mTOR, focal adhesion, and lysosome pathways are involved in autophagy, an immune mechanism for clearing intra-cellular proteins and organelles usually in response to cell stress (Figure 3E). ${ }^{28}$ Additionally, the systemic lupus erythematosus (SLE) pathway is highly enriched in the combination treatment. Given that SLE is an autoimmune disease, this finding is consistent with an immune-related functional effect. ${ }^{29}$ In conjunction with enrichment of hematopoietic cell lineage and CAMs signatures, perturbation of these additional pathways may provide further evidence of a molecular shift towards a gain of immune cell function.

\section{The addition of all-trans retinoic acid enhances GSK2879552 induced differentiation effects on acute myeloid leukemia cells}

Given that the GSK2879552 and ATRA combination affects hematopoiesis and cell adhesion molecule pathways, studies to confirm these results by measuring differentiation in AML cell lines were performed. Myeloid differentiation cell surface marker expression was measured by flow cytometry on 4 AML cell lines treated for two days with GSK2879552. Representative histograms of CD11b expression on MOLM-13 and OCI-AML3 cells treated with DMSO, 1000 nM GSK2879552, $100 \mathrm{nM}$ ATRA, or the combination of GSK2879552 and ATRA are shown (Figure 4A). In both cell lines, an increase in CD11b expression is evident with the combination. Cell lines were treated with a titration of GSK2879552 plus varying concentrations of ATRA. Percent of cells positive for $\mathrm{CD} 11 \mathrm{~b}$ were gated relative to isotype control and the values were plotted as dose response curves (Figure 4C). The increase in CD11b was dose responsive both with GSK2879552 and as increasing concentrations of ATRA were combined with GSK2879552 (Online Supplementary Table S3A). To determine whether the impact on CD11b expression with the combination is synergistic, an additivity threshold was calculated for each combination by adding together the increases obtained with each single agent and was compared to the observed values achieved with the combination (Figure 4D and Online Supplementary Figure S4A). MOLM-13 and SIG-M5 cells treated with as little as $1 \mathrm{nM}$ ATRA combined with GSK2879552 showed synergistic increases in CD11b. Synergistic effects were seen in OCI-AML3 cells with as little as 10 nM ATRA. THP-1 cells had a very robust response to GSK2879552 alone, reaching near $100 \%$ positive with as little as $12 \mathrm{nM}$ GSK2879552, precluding assessment of the combination effect (Online Supplementary Figure S4A).

The difference between the additive threshold and the actual percent of CD11b expression achieved was determined for each cell line (Figure 4B). Synergistic increases in CD11b expression were observed in three of four cell lines treated with the combination of ATRA and GSK2879552. Because GSK2879552 alone increased CD11b expression on nearly the entire population of THP-1 cells, median fluorescence intensity (MFI) values were determined to quantify shifts in CD11b expression (Online Supplementary Figure $S 4 B$ and C). Fold changes in CD11b MFI were determined relative to DMSO-treated control and additivity threshold values were calculated by adding together the fold increases obtained with each single agent. Actual increases in CD11b MFI were synergistic with as little as 1 nM ATRA combined with GSK2879552.

A hallmark of functional differentiation involves the appearance of morphological changes. ${ }^{30}$ To investigate the ability of the combination to elicit an altered cell appearance, MOLM-13 cells were treated for three days with DMSO, GSK-LSD1, ATRA, or the combination of GSKLSD1 plus ATRA. Morphology was assessed by May Grunwald/Giemsa stain (Online Supplementary Figure $S 4 D)$. The combination treatment resulted in the presence of lobular nuclei and granulocytic cytoplasm; both are characteristics of differentiated myeloid cells. These changes were largely absent from the cells treated with a single agent. These observations suggest that morphological differentiation represents a functional consequence of the gene expression and surface marker changes in AML cells caused by treatment with LSD1 inhibitor plus ATRA.

The combination of GSK2879552 and all-trans retinoic acid promotes caspase-mediated cell death

In addition to inhibiting the growth of cells (Figure 2), a decrease in cell number relative to the number of cells at the start of the assay was observed in the combination (Figure 5A). By day 3, in MOLM-13 cells, the combination treatment produced a negative growth death index (GDI) value that continued to decrease with additional duration of treatment. Neither ATRA nor GSK2879552 alone achieved negative GDI values. By day 5, in OCI-AML3 cells, GSK2879552 plus 1000 nM ATRA achieved a GDI value of $-50 \%$ compared to $3 \%$ with 1000 nM ATRA alone. A decrease in GDI value with the combination was observed in four of seven cell lines tested (Figure 5A and Online Supplementary Figure S5). These results indicate that, in addition to the improved inhibition of cell growth, AML cell death can be achieved with the combination of GSK2879552 and ATRA, and this cell death occurs at a time point after the observed increases in CD11b expression.

To confirm that the combination kills AML cells, and better understand the mechanism associated with this cytotoxic response, AML cells were treated with a dose titration of GSK2879552 plus and minus 1, 10, 100, and 
1000 nM ATRA for up to six days and caspase 3/7 induction was measured. Similar to the method used in analysis of the cell surface marker data (see above), on the day of peak caspase induction an additivity threshold was determined for each combination by adding the fold caspase induction values obtained with each individual treatment condition. By comparing the observed fold induction achieved to the additive threshold, synergistic caspase activation is revealed with the combination at ATRA concentrations as low as $100 \mathrm{nM}$ in MOLM-13 and OCIAML3 cells and as low as 10 nM ATRA in SIG-M5, MV-411 , and HL-60 cells (Figure 5C and Online Supplementary Figure S5B). Additionally, annexin V staining was performed on MOLM-13 cells treated for three days with GSK-LSD1 \pm 1000 nM ATRA. Annexin V positive cells increased with the combination to a greater extent than each compound alone, providing supporting evidence for the cell death caused by the combination (Online Supplementary Figure S5C). Importantly, the cells that are positive for Annexin V are also positive for CD11b, demonstrating that the cells that differentiate also undergo apoptosis (Online Supplementary Figure S5D).

Using an approach similar to that utilized in the cell surface marker assessment, the difference between the additive threshold of caspase activation and the observed value was determined for each cell line and a heatmap was generated (Figure 5B). The combination of GSK2879552 plus ATRA induced caspase 3/7 in five out of seven AML cell lines tested. Importantly, the caspase induction in the combination reached levels greater than 10 -fold above the additive threshold in all five of the positive cell lines at 100 nM ATRA, a concentration that corresponds to clinically achieved exposures. ${ }^{31,32}$

\section{Acute myeloid leukemia blast colony forming ability is impaired in patient-derived samples treated with a combination of GSK2879552 plus all-trans retinoic acid}

Translating a combination effect beyond cell lines is important in understanding the potential for success in clinical trials. To this end, we assessed GSK2879552 combined with 100 nM ATRA in blast cells derived from BM of nine different AML patients (Online Supplementary Supplementary Table S1A). Dose response curves for GSK2879552, ATRA, and the combination of GSK2879552 plus 100 nM ATRA are shown for two patients (Figure 6A and D). For patient AML0007, the maximum inhibition of blast colony formation achieved with GSK2879552 was 39\% while 100 nM ATRA had no effect on colony count. The combination of GSK2879552 and ATRA reached almost complete inhibition, a $>50 \%$ decrease in AML blast colony forming ability (Figure 6B). Similarly, the $\mathrm{IC}_{50}$ improved $>2$-fold from $105 \mathrm{nM}$ with GSK2879552 to $43 \mathrm{nM}$ with the combination (Figure 6C). A second patient-derived sample, $4031113 \mathrm{SH}$, had a modest improvement in maximum inhibition (54\% inhibition with GSK2879552, 4\% inhibition with ATRA, 69\% inhibition with the combination); however, the $\mathrm{IC}_{50}$ decreased $>10$ fold from $150 \mathrm{nM}$ with GSK2879552 to $11 \mathrm{nM}$ with the combination (Figure $6 \mathrm{E}$ and F). Overall, a combination effect was observed in seven out of nine patient samples analyzed in at least one of the two parameters measured (Online Supplementary Figure S6A and B). An increase in maximum inhibition of at least $15 \%$ with the combination was achieved in five out of eight samples and of at least $30 \%$ in three out of eight samples. One sample achieved $100 \%$ inhibition with GSK2879552 alone so an improvement in inhibition was not possible with the combination in this sample. A decrease in $\mathrm{IC}_{50}$ of at least 2 -fold with the combination was achieved in six out of nine samples and of at least 5-fold in two out of nine samples. Together, these data suggest that the combination effects are not limited to cell lines and that a lower dose of LSD1 inhibitor than is maximally efficacious as single agent may be sufficient to elicit the combination response.

\section{Discussion}

Acute myeloid leukemia is a deadly cancer characterized by accumulation of immature myeloid cells in the BM that undergo replication in an uncontrolled manner. Treatments such as chemotherapy focus on de-bulking the tumor by killing fast-growing cells. Unfortunately, it has been demonstrated that even after treatment some refractory cells remain that can resume growth resulting in disease relapse. ${ }^{33}$ A recent study has shown that inhibition of LSD1 can affect the leukemia-initiating population suggesting a potential clinical opportunity for LSD1 inhibitors in combination with de-bulking agents, even if these are not obviously beneficial as monotherapy. ${ }^{13}$

Another treatment option that has been more than $90 \%$ effective in APL involves the use of ATRA. ATRA has been shown to promote differentiation of myeloid cells and, therefore, the cells no longer possess replication potential. ${ }^{16}$ Applying this methodology more broadly to AML has thus far been unsuccessful because ATRA is unable to elicit a differentiation phenotype in non-APL AML. ${ }^{18}$ Interestingly, a new differentiation therapy, Enasidenib, was recently approved in the USA for the treatment of IDH2 mutant AML, demonstrating the potential of differentiation therapies in the non-APL AML setting. ${ }^{34}$

The results presented here indicate that features consistent with differentiation in AML cells can be achieved by inhibition of LSD1. Changes in cell surface expression of myeloid maturation markers is evident in all AML subtypes tested. LSD1 inhibition also increases superoxide anion production, indicative of a more granulocytic phenotype, in AML cells. These results combined with the decrease in blast cells of primary AML patient samples treated with GSK2879552 suggest the therapeutic opportunity for LSD1 inhibition in treating non-APL AML. The addition of ATRA synergistically enhanced the differentiation effect achieved with GSK2879552 through increased expression of myeloid cell surface marker CD11b. This is achieved at lower GSK2879552 concentrations than required when AML cells are treated without ATRA thus potentially widening the therapeutic index of LSD1 inhibition. Importantly this effect is observed across AML subtypes and is not exclusive to APL. Gene expression analysis revealed enrichment of the hematopoietic and CAM pathways further indicating that a primary mechanism in association with this combination involves differentiation. Pushing AML cells to differentiate with a combination of GSK2879552 and ATRA may offer profound implications for the success of AML treatment options for patients beyond APL or those with IDH2 mutations.

While enhancing differentiation provides an attractive rationale for pursuing the combination of LSD1 inhibitors with ATRA in the clinic, the observation that the combi- 
nation promotes cytotoxic response provides an additional rationale to explore this combination in AML patients. Treatment of non-APL AML cell lines with GSK2879552 and ATRA results in synergistic cleavage of caspases 3 and 7 , the executioner caspases. ${ }^{35}$ The synergistic cell death occurs after the observed changes in cell surface marker expression and may suggest that as cells reach terminal differentiation, this process is a signal to the cells to undergo apoptosis in a manner similar to the life cycle of normal immune cells. ${ }^{36}$ Given that the gene set enrichment analysis revealed that the lysosome and MTOR pathways are also affected with the combination, another possibility is that the differentiation triggers an autophagic response that leads to a switch to apoptosis. ${ }^{28}$ Importantly, applying the combination to primary patient samples ex vivo successfully illustrates that a beneficial combination effect is not restricted to cultured cell lines. The broad response of cell lines and primary AML samples to the combination does preclude the ability to identify a biomarker preclinically. Pretreatment biopsy assessment will, therefore, be required to determine which patients will most likely respond to the combination. Taken together, these results highlight the possibility of achieving a cure in AML through treatment with LSD1 inhibitors and ATRA.

\section{References}

1. Fang J, Ying $\mathrm{H}$, Mao $\mathrm{T}$, et al. Upregulation of CD11b and CD86 through LSD1 inhibition promotes myeloid differentiation and suppresses cell proliferation in human monocytic leukemia cells. Oncotarget. 2017;8(49):85085-85101.

2. Arber DA, Orazi A, Hasseriian R, et al. The 2016 revision to the World Health Organization classification of myeloid neoplasms and acute leukemia. Blood. 2016; 127(20):2391-2405.

3. Dombret H, Gardin C. An update of current treatments for adult acute myeloid leukemia. Blood. 2016;127(1):53-61.

4. Wouters BJ, Delwel R. Epigenetics and approaches to targeted epigenetic therapy in acute myeloid leukemia. Blood. 2016; 127(1):42-52.

5. Zhou C, Wu F, Lu L, et al. Structure activity relationship and modeling studies of inhibitors of lysine specific demethylase 1 . PLoS One. 2017;12(2):e0170301.

6. Shi YJ, Matson C, Lan F, Iwase S, Baba T, Shi Y. Regulation of LSD1 histone demethylase activity by its associated factors. Mol Cell. 2005;19(6):857-864.

7. Saleque S, Kim J, Rooke HM, Orkin SH. Epigenetic regulation of hematopoietic differentiation by Gfi- 1 and Gfi- $1 \mathrm{~b}$ is mediated by the cofactors CoREST and LSD1. Mol Cell. 2007;27(4):562-572.

8. Harris WJ, Huang X, Lynch JT, et al. The histone demethylase KDM1A sustains the oncogenic potential of MLL-AF9 leukemia stem cells. Cancer Cell. 2012;21(4):473-487.

9. McGrath JP, Williamson KE, Balasubramanian S, et al. Pharmacological Inhibition of the Histone Lysine Demethylase KDM1A Suppresses the Growth of Multiple Acute Myeloid Leukemia Subtypes. Cancer Res. 2016;76(7):1975-1988.

10. Lynch JT, Harris WJ, Somervaille TC. LSD1 inhibition: a therapeutic strategy in cancer? Expert Opin Ther Targets. 2012;16(12):12391249.

11. Mohammad HP, Smitheman KN, Kamat $\mathrm{CD}$, et al. A DNA Hypomethylation Signature Predicts Antitumor Activity of LSD1 Inhibitors in SCLC. Cancer Cell. 2015;28(1):57-69

12. Maes T, Mascaro C, Tirapu I, et al. ORY-
1001, a Potent and Selective Covalent KDM1A Inhibitor, for the Treatment of Acute Leukemia. Cancer Cell. 2018; 33(3):495-511 e412

13. Cusan M, Cai SF, Mohammad HP, et al. LSD1 inhibition exerts its antileukemic effect by recommissioning PU.1- and C/EBPalpha-dependent enhancers in AML Blood. 2018;131(15):1730-1742.

14. de The H, Pandolfi PP, Chen Z. Acute Promyelocytic Leukemia: A Paradigm for Oncoprotein-Targeted Cure. Cancer Cell. 2017;32(5):552-560

15. Al Tanoury Z, Piskunov A, Rochette-Egly C. Vitamin $\mathrm{A}$ and retinoid signaling: genomic and nongenomic effects. J Lipid Res. 2013:54(7):1761-1775.

16. Melnick A, Licht JD. Deconstructing a disease: RARalpha, its fusion partners, and their roles in the pathogenesis of acute promyelocytic leukemia. Blood. 1999; 93(10):3167-3215.

17. Zhou GB, Zhao WL, Wang ZY, Chen SJ, Chen $Z$. Retinoic acid and arsenic for treating acute promyelocytic leukemia. PLoS Med. 2005;2(1):e12

18. Johnson DE, Redner RL. An ATRActive future for differentiation therapy in AML. Blood Rev. 2015;29(4):263-268.

19. Schenk T, Chen WC, Gollner S, et al. Inhibition of the LSD1 (KDM1A) demethylase reactivates the all-trans-retinoic acid differentiation pathway in acute myeloid leukemia. Nat Med. 2012;18(4):605-611.

20. Kaniskan HU, Martini ML, Jin J. Inhibitors of Protein Methyltransferases and Demethylases. Chem Rev. 2018; 118(3):9891068.

21. Foucquier J, Guedj M. Analysis of drug combinations: current methodological landscape. Pharmacol Res Perspect. 2015 3(3):e00149.

22. Sprussel A, Schulte JH, Weber S, et al. Lysine-specific demethylase 1 restricts hematopoietic progenitor proliferation and is essential for terminal differentiation. Leukemia. 2012;26(9):2039-2051.

23. van Lochem EG, van der Velden VH, Wind HK, te Marvelde JG, Westerdaal NA, van Dongen JJ. Immunophenotypic differentiation patterns of normal hematopoiesis in human bone marrow: reference patterns for age-related changes and disease-induced shifts. Cytometry B Clin Cytom.
2004:60(1):1-13

24. Chen L, Flies DB. Molecular mechanisms of $\mathrm{T}$ cell co-stimulation and co-inhibition. Nat Rev Immunol. 2013;13(4):227-242.

25. Marsee DK, Pinkus GS, Yu H. CD71 (transferrin receptor): an effective marker for erythroid precursors in bone marrow biopsy specimens. Am J Clin Pathol. 2010; 134(3):429-435

26. Song HO, Ryu JS. Superoxide anion production by human neutrophils activated by Trichomonas vaginalis. Korean J Parasitol. 2013;51(4):479-484.

27. Finkel TH, Pabst MJ, Suzuki H, et al. Priming of neutrophils and macrophages for enhanced release of superoxide anion by the calcium ionophore ionomycin. Implications for regulation of the respiratory burst. J Biol Chem. 1987;262(26):12589-12596.

28. Marino G, Niso-Santano M, Baehrecke EH Kroemer G. Self-consumption: the interplay of autophagy and apoptosis. Nat Rev Mol Cell Biol. 2014;15(2):81-94.

29. Danchenko N, Satia JA, Anthony MS Epidemiology of systemic lupus erythematosus: a comparison of worldwide disease burden. Lupus. 2006;15(5):308-318

30. Grassi L, Pourfarzad F, Ullich S, et al. Dynamics of Transcription Regulation in Human Bone Marrow Myeloid Differentiation to Mature Blood Neutrophils. Cell Rep. 2018;24(10):27842794

31. Regazzi MB, Iacona I, Gervasutti C Lazzarino M, Toma S. Clinical pharmacokinetics of tretinoin. Clin Pharmacokinet. 1997;32(5):382-402.

32. Russo D, Regazzi M, Sacchi S, et al. All-trans retinoic acid (ATRA) in patients with chronic myeloid leukemia in the chronic phase. Leukemia. 1998;12(4):449-454.

33. Hourigan CS, Gale RP, Gormley NJ Ossenkoppele GJ, Walter RB. Measurable residual disease testing in acute myeloid leukaemia. Leukemia. 2017;31(7):14821490.

34. Kim ES. Enasidenib: First Global Approval. Drugs. 2017;77(15):1705-1711.

35. Koff JL, Ramachandiran S, Bernal-Mizrachi L. A time to kill: targeting apoptosis in cancer. Int J Mol Sci. 2015;16(2):2942-2955.

36. Nagata S. Apoptosis and clearance of apoptotic cells. Annu Rev Immunol. 2018; 36:489-517. 